\title{
Gold Palladium Thin Films: Multi-twinned Nanoparticles to Five-fold Annealing Twins
}

\author{
Prakash Parajuli $^{1}$, Arturo Ponce $^{1 *}$ \\ 1. University of Texas at San Antonio, San Antonio, Texas, USA. \\ * Corresponding author: Arturo.ponce@utsa.edu
}

Nanocrystals (nanoparticles and nanocrystalline thin films) are the key component of nanotechnology. In the last 50 years, numerous approaches to produce nanocrystals have been reported using both chemical and physical means. The chemical route is common in the colloidal synthesis of nanoparticles whereas physical vapor deposition, a relatively simple technique was used to deposit metals on alkali halides starting from the mid-nineteenth century. Since then various important research works were carried out on epitaxial growth of metallic thin films evaporated on alkali halide surfaces [1-3]. Still, some key features such as the precise mechanism controlling the particle-substrate orientation, and the influence of the size, shape and relative orientation of the evaporated particles on the microstructure of the films and finally the performance of the materials are under investigation [2].

Various nanoscale twin structures such as lamellar twin, two fold twin and multi-fold twins are commonly observed in low stacking fault fcc materials like gold and copper, and play an important role in strengthening nanocrystalline materials without significant loss of ductility $[4,5]$. Among them, five-fold twins has received considerable attention recently, not only due to their role in increasing the yield strength and elastic modulus of the nanocrystalline metals $[6,7]$ but also because of the lack of clear understanding of their formation mechanism [8-10]. It is believed that five-fold deformation twins (FDTs) are formed by the sequential emission of partial dislocations from the GBs (GBs) due to high external stress [8] but the formation mechanism for five-fold annealing twins are still in debate [9, 10].

Herein, vacuum deposited $\mathrm{Au} / \mathrm{Pd}$ thin film $\left(\mathrm{Au}_{93} \mathrm{Pd}_{7}\right)$ is explored; structural analysis of nanoparticles corresponding to $10 \AA$ film and microstructure and five-fold annealing twin of $350 \AA$ continuous film. Thin films were deposited under high vacuum at the substrate temperature of $473 \mathrm{~K}$, the continuous film was annealed for 20 minutes after deposition. Atomic resolution characterization was carried out in JEOL ARM 200F using Cs aberration corrected scanning transmission electron microscopy using High angle annular dark field (STEM-HAADF) detector and diffraction patterns were acquired using JEOL TEM 2010F. Furthermore, geometric phase analysis (GPA) were carried out over the five-fold annealing twin to determine the strain distribution across the various segments of the five-fold.

Summary of the $\mathrm{Au} / \mathrm{Pd}$ nanoparticles corresponding to $10 \AA$ film is shown in Figure $1(\mathrm{a}-\mathrm{b})$. Results shows basically two types of nanoparticles: twinned (decahedral, icosahedral and single twinned) and regular fcc (truncated octahedral and bipyramidal). Majority (almost 85\%) of the particles are twinned. Similar distribution of particles were reported in vacuum deposited gold nanoparticles under similar deposition conditions [3]. Atomic-resolution images of two representative particles (multitwinned and truncated bipyramidal) is shown in Figure 1(b). Low magnified electron micrograph of the continuous film is shown in Figure 1(c) (Inset diffraction patterns). Many twin structures (lamellar, two-fold twins and multifold twins) were revealed in the film. Atomic-resolution image of one of the five-fold twin and geometrical phase analysis across the five-fold junction is shown in the figure 1(d) showing compressive (blue color) and tensile (red color) shear zone. Measured shear-strain profiles across the segments are shown in Figure 
1(d). As illustrated in figures, it can be seen that, strain profiles fluctuate randomly around $+1.2 \%$ (tensile) to $-2.5 \%$ (compressive) across different segments of five-fold structures. These results show that the subunits of the AuPd five-fold structures are not a relaxed FCC crystal, but each subunit is stressed, and the strain is distributed non-uniformly. Detailed analyses of the various types of five-fold twin structures are illustrated in other report $[11,12]$.

\section{References:}

[1] S Ino, D Watanabe and S Ogawa, J Phys Soc Jpn 19 (1964), p. 881.

[2] R Mendoza-Cruz et al., Crystal Growth \& Design 17 (2017), p. 6062.

[3] P Parajuli et al., Cryst Res Technol 53 (2018), p. 1.

[4] D Bufford et al., Nat Commun 5 (2014), p. 4864.

[5] K Lu, L Lu and S Suresh, Science 324 (2009), p. 349.

[6] JY Wu et al., Nano Lett 11 (2011), p. 5264.

[7] F Niekiel, E Spiecker and E Bitzek, J Mech Phys Solids 84 (2015), p. 358.

[8] Y Zhu, X Liao and R Valiev, Appl Phys Lett 86 (2005), p. 103112.

[9] E Bringa et al., Scripta Mater 59 (2008), p. 1267.

[10] SL Thomas, AH King and DJ Srolovitz, Acta Mater 113 (2016), p. 301.

[11] P Parajuli et al., Mater Lett 244 (2019), p. 88.

[12] The authors acknowledge funding from the Welch foundation (grant no. AX-1615).
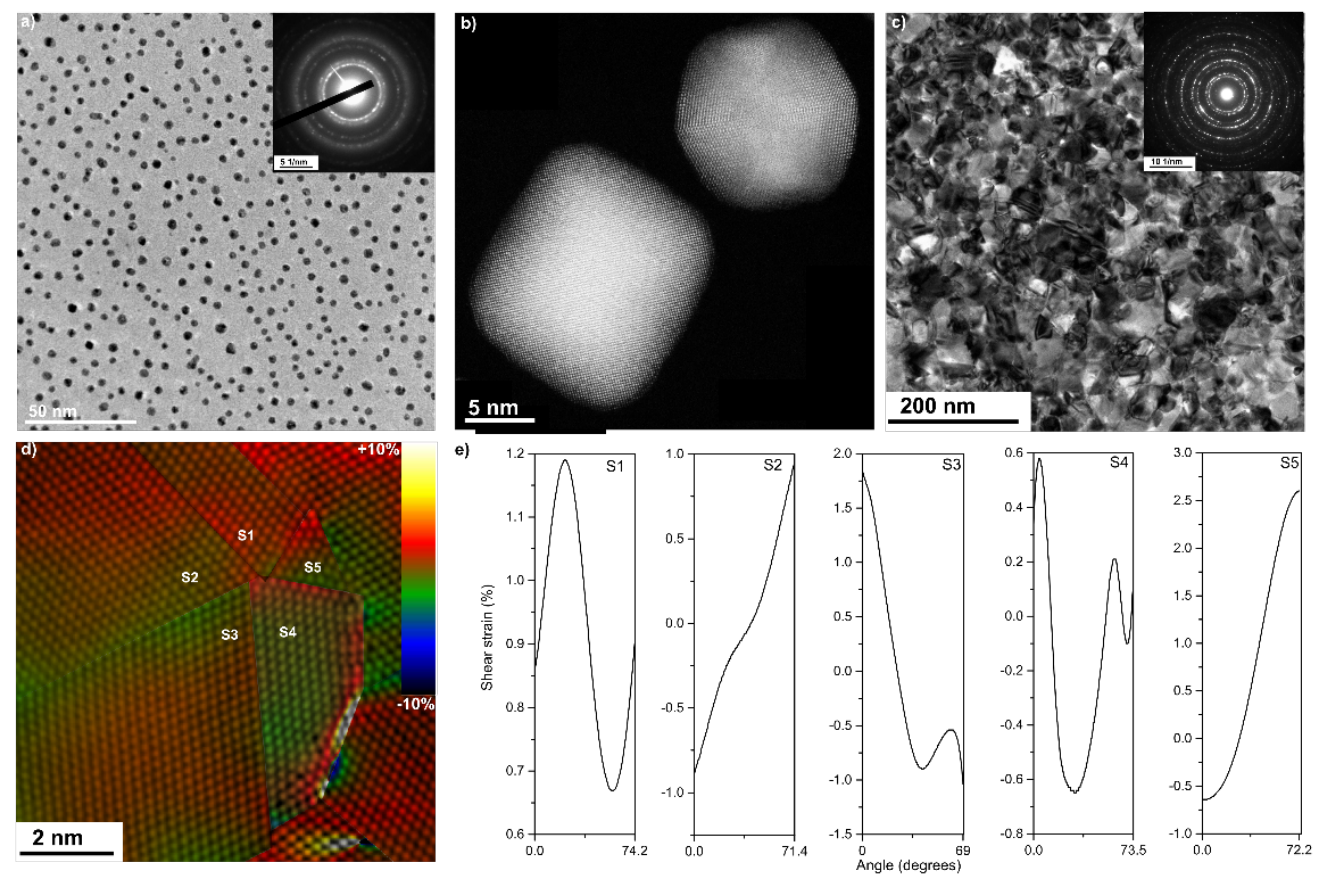

Figure 1. $\mathrm{Au} / \mathrm{Pd}$ thin films. a) Distribution of $\mathrm{Au} / \mathrm{Pd}$ nanoparticles in $10 \AA$ film (inset diffraction patterns) b) STEM-HAADF images of icosahedral and truncated bipyramidal particles c) Electron micrograph of continuous ( $350 \AA$ ) film (inset diffraction patterns) d) Overlapped STEM-HAADF image and strain maps of five-fold twin, and e) Strain profiles along different segments of the fivefold twin. Polycrystalline nature of particles and films and non-uniform distribution of strain across the segments of five-fold twin is shown in the figure. 